Nachhaltigkeitsberichterstattung in Deutschland

\section{Ergebnisse des \\ IÖW-future-Rankings 2007}

Deutschlands Großunternehmen berichten vermehrt und vielfältig über ihre gesellschaftliche Verantwortung. Das diesjährige Ranking bewertete 58 aktuelle Nachhaltigkeits- und Umweltberichte. Otto ging daraus als Sieger hervor, gefolgt von RWE und BASF. Von Jana Gebauer

D as Ranking der Nachhaltigkeitsberichte vom Institut für ökologische Wirtschaftsforschung (IÖW) und vom Unternehmensverband future e.V. bewertet die inhaltliche und kommunikative Qualität der gesellschaftsbezogenen Berichterstattung der 150 größten deutschen Unternehmen (1). 1994 als Pioniere mit einem Ranking der Umweltberichte gestartet, gingen IÖW und future in diesem Jahr in die sechste Runde. Seit der Umstellung auf das breite Spektrum nachhaltigkeitsbezogener Anforderungen im Jahr 2005 liegt der Berichtsbewertung ein anspruchsvolles Set von 48 sozialen, ökologischen, ökonomischen sowie kommunikativen Kriterien zugrunde (2). Das Kriterienset und die Ergebnisberichte der Rankings 2005 und 2007 sind auf der Pro-

jekt-Website veröffentlicht (3). Im Folgenden werden ausgewählte Resultate des aktuellen Rankings präsentiert.

Die 150 größten deutschen Unternehmen wurden auf Basis der Statistik der Frankfurter Allgemeinen Zeitung vom April 2007 bestimmt und in das Ranking einbezogen (4). Diese verteilen sich auf 15 Branchen, von A wie Automobil bis V wie Versicherungen, wie Tabelle 1 zeigt. 39 dieser Unternehmen berichten derzeit überhaupt nicht über ihre gesellschaftliche Verantwortung, weder integriert in den Geschäftsbericht noch im Internet. Der größte Anteil dieser sogenannten Non-Reporter findet sich bei den Versicherern, den Elektrotechnikherstellern und im Handel. 30 Unternehmen können als Tochterunternehmen auf einen

Tabelle 1: Branchenübersicht zum Ranking 2007

\begin{tabular}{cclccll}
\hline \multicolumn{2}{c}{$\begin{array}{l}\text { Rang nach } \\
\text { Punkten }\end{array}$} & Branche & \multicolumn{2}{c}{ Anzahl } & Ø Punkte & Branchensieger \\
2007 & 2005 & & Unter- & Be- & Branche & \\
\hline 1 & 8 & Bau & 4 & 1 & 431 & HochTief AG \\
\hline 2 & 1 & Handel/Bekleidung & 20 & 5 & 407 & OTTO GmbH \& Co.KG \\
\hline 3 & 3 & Elektro/Technik & 21 & 3 & 391 & Robert Bosch GmbH \\
\hline 4 & 2 & Automobil & 7 & 4 & 366 & Volkswagen AG \\
\hline 5 & 5 & Papier/Druck/Verlage & 3 & 2 & 366 & Axel Springer AG \\
\hline 6 & 4 & Transport/Logistik & 6 & 4 & 362 & Deutsche Bahn AG \\
\hline 6 & 7 & Energie/Versorger & 16 & 5 & 355 & RWE AG \\
\hline 8 & 4 & Telekommunikation & 5 & 3 & 348 & Vodafone D2 GmbH \\
\hline 9 & 6 & Banken & 15 & 7 & 313 & WestLB \\
\hline 10 & 13 & Grundstoffe & 8 & 4 & 301 & HeidelbergCement AG \\
\hline 11 & 9 & Chemie/Pharma & 17 & 13 & 298 & BASF AG \\
\hline 11 & 12 & Maschinenbau & 6 & 3 & 252 & Linde AG \\
\hline 13 & 11 & Versicherungen & 15 & 2 & 241 & Münchener Rück- \\
& & & & & & versicherungsgesellschaft \\
\hline 14 & 10 & Nahrung/Genuss & 5 & 1 & 197 & Südzucker AG \\
\hline 15 & 14 & Mischkonzerne & 2 & 1 & 135 & Procter\&Gamble \\
& & & & & & Service GmbH \\
\hline
\end{tabular}

Quelle: Eigene Darstellung
Bericht der ausländischen Mutter verweisen. Weitere 23 berichten knapp im Geschäftsbericht oder im Internet, in standort- oder themenbezogenen Broschüren. Damit bleiben unter den 150 größten deutschen Unternehmen 58, die einen eigenständigen Umwelt-, Nachhaltigkeits-, CSR- oder vergleichbaren Bericht veröffentlichen, davon vier Unternehmen erstmals (5). Die meisten Berichte kommen von den Chemie- und Pharmaunternehmen sowie den Banken. 2005 lag die Zahl der Reporter bei 53, die der Non-Reporter bei 42. Aufgrund der starken Dynamik in den Top 150 hat ein bloßer Vergleich der Gesamtzahlen jedoch nur begrenzte Aussagekraft für die Entwicklung der Nachhaltigkeitsberichterstattung.

Der beste Bericht des diesjährigen Rankings erzielte 543 Punkte, knapp 50 Punkte mehr als der Sieger 2005. Der Durchschnittswert aller Berichte ist dabei um 11 auf 336 Punkte gestiegen; allerdings hat sich die Schere zwischen Erstund Letztplatziertem weiter geöffnet. Auch in den Einzelplatzierungen der Unternehmen haben sich starke, positive wie negative, Veränderungen ergeben, wie Tabelle 2 verdeutlicht. Im Ergebnisbericht gehen wir genauer auf mögliche Ursachen ein, die sowohl in der Berichterstattung der Unternehmen als auch in der Methodik des Rankings liegen.

Das Ranking 2005 hatte den stärksten Entwicklungsbedarf in der Berichterstattung zu sozialen Anforderungen identifiziert; dies gilt weiterhin auch 2007. Die Branchenleistungen fallen hier stark auseinander. Die Top-Berichte kommen aus den Branchen Telekommunikation, Energieversorger und Handel. Sie schaffen es, zu Aspekten wie Chancengleichheit, Arbeitnehmerrechte oder Gleichstellung über reine Bekenntnisse hinaus konkrete Umsetzungsmaßnahmen abzubilden. Allgemeines Lieblingsthema im Bereich Gleichstellung ist übrigens die Bereitstellung von Kinderbetreuungsplätzen, als Programm zur gezielten Frauenförderung ein eher eingeschränkter Fokus. Eindeutig an Relevanz gewonnen hat das Thema Antikorruption; selten fehlt der Verweis auf einen Verhaltenskodex oder zumindest dessen Entwicklung. Wirklich 
gute Berichterstattung heißt hier jedoch, konkrete Korruptionsrisiken und -fälle zu benennen und systematische Präventivund Sanktionsmaßnahmen darzustellen. Zunehmend finden Angaben zu Steuerzahlungen, eine wichtige unternehmerische Leistung für die Gesellschaft, Eingang in die Berichterstattung. Leider lässt sich Gleiches noch nicht zu den von der Gesellschaft erhaltenen Leistungen wie Fördermittel und Subventionen sagen.

Über Maßnahmen und Leistungen im Bereich ökologischer Anforderungen zu berichten, ist für die Unternehmen dageauch hier das Gesamtbild nicht weiter verbessert. Die Branchenleistungen unterscheiden sich dabei kaum; als Bessere unter Gleichen können Elektro-, Automobilund Verlagsunternehmen gelten. Sie zeichnet eine solide und systematische Berichterstattung zu den Umwelt-Klassikern Produktentwicklung, Emissionen und Abfallmanagement aus. Kein Klassiker, vielmehr immer noch bedrohte Art ist das Thema Naturschutz und Biodiversität. Die Verbesserung der Berichterstattung um 50 Prozent ist zwar enorm, bezieht sich jedoch auf ein niedriges Ausgangsniveau und wurde vor allem durch herausragende Einzelleistungen von Verlagen, gen selbstverständlich. Allerdings hat sich

Energieversorgern und Transportunternehmen erreicht.

Die Berichterstattung zu Fragen der Prozesssicherheit wurde dagegen allgemein deutlich ausgebaut und zwar sowohl mit Blick auf mögliche Gesundheitsgefahren als auch hinsichtlich resultierender Umweltbelastungen. Die Darstellungen im Bereich Arbeitssicherheit und Gesundheitsschutz sowie zum chemiespezifischen Kriterium der Produktions- und Transportunfälle sind in der Regel umfassend, sehr konkret und nachvollziehbar.

Die Betrachtung der Lieferkette, 2005 bereits als ausbaubedürftig identifiziert, weist weiterhin große Lücken auf. Dies gilt sowohl in sozialer Hinsicht auf Arbeitsbedingungen und Geschäftsbeziehungen als auch bezüglich der Umweltaspekte. Gesellschaftliche Verantwortung wird von den meisten Unternehmen noch stark auf die eigene Wertschöpfungsstufe bezogen; eine Ausnahme bilden die Handelsunternehmen, die in der vorgelagerten Lieferkette einen wesentlichen Verantwortungsbereich für sich sehen.

Bei den sogenannten integrierten Anforderungen, die sich auf den Managementbereich und formale Aspekte beziehen, lässt sich wie schon 2005 Folgendes

Tabelle 2: Top 20 des Rankings 2007

\begin{tabular}{|c|c|c|c|c|c|c|c|}
\hline \multirow{2}{*}{\multicolumn{2}{|c|}{$\begin{array}{l}\text { Rang nach } \\
\text { Punkten }\end{array}$}} & \multirow[t]{3}{*}{ Unternehmen } & \multirow[t]{3}{*}{ Berichtsart } & \multicolumn{4}{|c|}{ Punktzahl } \\
\hline & & & & \multirow{2}{*}{ Gesamt } & \multirow{2}{*}{ sozial } & \multirow{2}{*}{$\begin{array}{l}\text { ökolo- } \\
\text { gisch }\end{array}$} & \multirow{2}{*}{ integriert } \\
\hline & & & & & & & \\
\hline 1 & 3 & OTTO GmbH \& Co. KG & Nachhaltigkeitsbericht & 543 & 153 & 135 & 255 \\
\hline 2 & 6 & RWE AG & Nachhaltigkeitsbericht & 528 & 132 & 126 & 270 \\
\hline 3 & 13 & BASF AG & Unternehmensbericht & 497 & 140 & 117 & 240 \\
\hline 4 & 2 & $\begin{array}{l}\text { KarstadtQuelle AG } \\
\text { (Arcandor AG) }\end{array}$ & Nachhaltigkeitsbericht & 478 & 103 & 129 & 246 \\
\hline 5 & - & WestLB AG & Nachhaltigkeitsbericht & 476 & 110 & 144 & 222 \\
\hline 6 & 19 & Wacker Chemie AG & Nachhaltigkeitsbericht & 470 & 115 & 105 & 250 \\
\hline 7 & 31 & Bayer AG & Nachhaltigkeitsbericht & 464 & 126 & 126 & 212 \\
\hline 8 & 4 & Axel Springer AG & Nachhaltigkeitsbericht & 439 & 106 & 139 & 194 \\
\hline 9 & 7 & Volkswagen AG & Nachhaltigkeitsbericht & 436 & 99 & 113 & 224 \\
\hline 10 & 9 & BMW Group & Nachhaltigkeitsbericht & 431 & 78 & 138 & 215 \\
\hline 10 & 26 & HochTief AG & Nachhaltigkeitsbericht & 431 & 140 & 104 & 187 \\
\hline 12 & - & Deutsche Bahn AG & Nachhaltigkeitsbericht & 428 & 108 & 138 & 182 \\
\hline 12 & 26 & Merck KGaA & CSR-Bericht & 428 & 92 & 105 & 231 \\
\hline 14 & 1 & Henkel KGaA & Nachhaltigkeitsbericht & 426 & 101 & 117 & 208 \\
\hline 15 & 12 & Robert Bosch GmbH & CSR-Bericht & 424 & 113 & 120 & 191 \\
\hline 16 & - & Vodafone D2 GmbH & CSR-Bericht & 414 & 131 & 68 & 215 \\
\hline 17 & 5 & adidas AG & Sozial- und Umweltbericht & 413 & 73 & 100 & 240 \\
\hline 17 & 15 & Degussa AG (Evonik) & CSR-Bericht & 413 & 95 & 108 & 210 \\
\hline 19 & 10 & $\begin{array}{l}\text { BSH Bosch und Siemens } \\
\text { Hausgeräte GmbH }\end{array}$ & Nachhaltigkeitsbericht & 402 & 82 & 120 & 200 \\
\hline 20 & - & E.ON AG & CSR-Bericht & 398 & 102 & 96 & 200 \\
\hline
\end{tabular}

Quelle: Eigene Darstellung beobachten. Die Unternehmen zeigen Vision und Verbindlichkeit im Umgang mit ihrer gesellschaftlichen Verantwortung, verfügen häufig über die relevanten Managementsysteme und unterziehen sich zumindest internen Auditierungen. Sie scheuen jedoch weiter die kritischen Fragen zum Stand der Zielerreichung und zu Zielkonflikten, zu zentralen Herausforderungen und Umsetzungsproblemen. Hierzu finden nur wenige Unternehmen klare und überprüfbare Formulierungen.

Welche weiteren Eindrücke liefert das Ranking 2007? Wohl vor allem den, dass sich die Nachhaltigkeitsberichterstattung zunehmend ausdifferenziert. Die Berichte sind wie Bücher dick oder broschürenschmal, FSC-gelabelt, hochglanzgedruckt oder virtuell. Sie arbeiten stark mit Gestaltung und Wort oder bleiben geschäftlich, sachlich und nüchtern; sie konzentrieren sich auf Schwerpunkte und Interessengruppen oder setzen auf Vollständigkeit über die Breite des Kriteriensets. Reine Umweltberichterstattung gibt es übrigens kaum noch. Im Ganzen heißt das wohl: Es bleibt spannend und die Bewertung eine Herausforderung. Für 2009.

\section{Anmerkungen}

(1) Das Ranking 2007 wird durch die Förderung des Rates für Nachhaltige Entwicklung und aus Eigenmitteln finanziert.

(2) Die Kriterien gehen mit unterschiedlicher Gewichtung in die Bewertung ein; ihre Erfüllung wird mit 0 bis 5 Punkten bewertet. Maximal sind 700 Punkte zu erreichen.

(3) Projektwebsite des Rankings: www.rankingnachhaltigkeitsberichte.de

(4) Für das Ranking werden die jeweils größten 100 Industrie- und Dienstleistungsunternehmen, je 15 Versicherer und Banken sowie 20 Handelsunternehmen zusammengefasst.

(5) Relevant ist hier der aktuelle deutsche gedruckte Bericht beziehungsweise ein stichtagsbezogenes PDF des Internetauftritts aus den Jahren 2005 bis 2007.

\section{AUTORIN + KONTAKT}

Jana Gebauer ist wissenschaftliche Mitarbeiterin im Forschungsfeld Ökologische Unternehmenspolitik am Institut für ökologische Wirtschaftsforschung (IÖW).

Institut für ökologische Wirtschaftsforschung (IÖW), Potsdamer Str. 105, 10785 Berlin. Tel.: +4930 884594-0,

E-Mail: jana.gebauer@ioew.de, Ranking-Website: www.ranking-nachhaltigkeitsberichte.de 
(c) 20I0 Authors; licensee IÖW and oekom verlag. This is an article distributed under the terms of the Creative Commons Attribution Non-Commercial No Derivates License (http://creativecommons.org/licenses/by-nc-nd/3.o/), which permits unrestricted use, distribution, and reproduction in any medium, provided the original work is properly cited. 